\title{
The lung microbiome: clinical and therapeutic implications
}

\author{
Alessio Fabbrizzi ${ }^{1} \cdot$ Amedeo Amedei $^{1,3} \cdot$ Federico Lavorini $^{1} \cdot$ Teresa Renda $^{2} \cdot$ Giovanni Fontana $^{1}$
}

Received: 19 July 2019 / Accepted: 27 September 2019 / Published online: 31 October 2019

(c) Società Italiana di Medicina Interna (SIMI) 2019

\begin{abstract}
The human respiratory tract, usually considered sterile, is currently being investigated for human-associated microbial communities. According to Dickson's conceptual model, the lung microbiota (LMt) is a dynamic ecosystem, whose composition, in healthy lungs, is likely to reflect microbial migration, reproduction, and elimination. However, which microbial genera constitutes a "healthy microbiome" per se remains hotly debated. It is now widely accepted that a bi-directional gut-lung axis connects the intestinal with the pulmonary microbiota and that the diet could have a role in modulating both microbiotas as in health as in pathological status. The LMt is altered in numerous respiratory disorders such as obstructive airway diseases, interstitial lung diseases, infections, and lung cancer. Some authors hypothesize that the use of specific bacterial strains, termed "probiotics," with positive effects on the host immunity and/or against pathogens, could have beneficial effects in the treatment of intestinal disorders and pulmonary diseases. In this manuscript, we have reviewed the literature available on the LMt to delineate and discuss the potential relationship between composition alterations of LMt and lung diseases. Finally, we have reported some meaningful clinical studies that used integrated probiotics' treatments to contrast some lung-correlated disorders.
\end{abstract}

Keywords Lung microbiota $\cdot$ Microbiota $\cdot$ Microbiome $\cdot$ Gut-lung axis $\cdot$ Dysbiosis $\cdot$ Probiotics

\section{Introduction}

The term microbiome was first used by Lederberg and McCray "to identify the ecological community of commensal, symbiotic, and pathogenic microorganisms that literally share our body space" [1]. In 2015, based on the concept of "biome", i.e., the biotic and abiotic factors of a given environment, Marchesi and Ravel, defined the microbiome as the entire "habitat" that is comprised of the microorganisms (bacteria, archaea, lower and higher eukaryotes, and viruses), their genomes (i.e., genes), and the surrounding environmental conditions [2]. Humans co-evolved with

Amedeo Amedei

aamedei@unifi.it

1 Department of Clinical and Experimental Medicine, University of Florence, Largo Brambilla 3, 50134 Florence, Italy

2 Respiratory Unit, Careggi University Hospital, Florence, Italy

3 Sod of Interdisciplinary Internal Medicine, Azienda Ospedaliera Universitaria Careggi (AOUC), University of Florence, 50134 Florence, Italy microbes in the environment and each body habitat has a unique set of microorganisms in its microbiota, which is established within the first 1-3 years of life and remains relatively stable throughout the life span [3]. Residential microbes perform metabolic functions and are involved in host functions such as defence, metabolism, and reproduction [4]. The microbiota usually was defined using molecular methods relying predominantly on the analysis of $16 \mathrm{~S}$ rRNA genes, 18S rRNA genes, or other marker genes and genomic regions, amplified and sequenced from given biological samples [2]. Taxonomic assignments can be performed using a variety of tools that assign each sequence to a microbial taxon (bacteria, archaea, or lower eukaryotes) at different taxonomic levels according to phyla, classes, orders, families, genera, and species [2]. In each body district, only a few phyla are represented, accounting for hundreds of bacterial species [5] (Fig. 1). Conserved genes are a target that is used to study the microbiome, particularly in bacteria. At present, the most used molecular method to study the microbiome is sequencing of regions of a conserved gene, such as the hypervariable regions of the $16 \mathrm{~S}$ ribosomal RNA gene [6]. Other higher resolution methods for microbiota profiling are currently being used to disclose the functional link between 


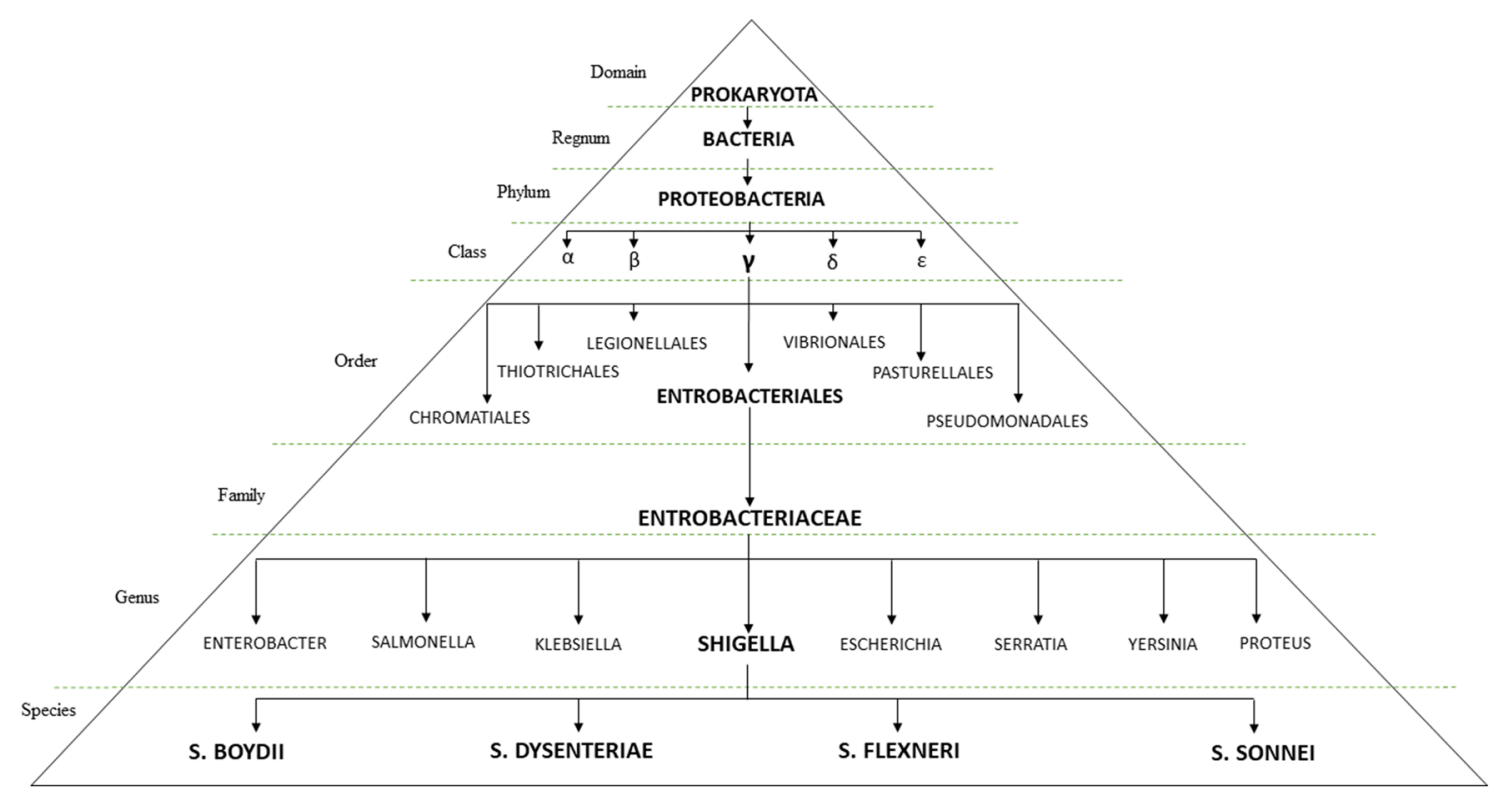

Fig. 1 Taxonomic classification: example of Shigella genus

the lungs and its microbiota [7]. The growing interest in the relationship between the microbial population and the human body accounts for the large amount of published papers on the topic in recent years [8-12].

This paper aims to provide an overview of the lung microbiota (LMt); more specifically, we will describe the possible LMt role in the genesis of some of the most epidemiologically relevant respiratory disorders in humans, particularly smoke-related diseases. We have also attempted at delineating the potential therapeutic implications of the relationship between the lungs and associated microbiota.

\section{Lung microbiota}

Although originally believed to be sterile, the lung shows a microbiota that varies in both physiological and pathological conditions [13]. Furthermore, bacteria, fungi, viruses, and their interactions may all be important in lung health and in the development of respiratory diseases [14]. The finding that a unique lung harbours a unique microbiota, irrespective of health or disease, has prompted a wealth of research to not only categorise the distinct microbiota of the healthy lung, but also that of the sickly lung [14]. The healthy human lung contains a variety of commensal microorganisms, especially bacteria, that can show substantial heterogeneity between individuals and across the different lung regions [15]. The LMt composition differs significantly between the upper and lower respiratory tracts in healthy individuals, enquiring if samples of the upper airways can reflect the microbiome in the lower respiratory tract [16]. The prevalence of distinct bacterial species in these compartments suggests the hypothesis of niche-specific microbial colonization at distinct anatomical sites [17]. Many authors agreed that the healthy LMt has a low density of microbial populations, mainly represented by phyla such as Firmicutes, Bacteriodetes, Proteobacteria, Fusobacteria, and Actinobacteria [18]. Mathieu et al. [19] hypothesized that small numbers of bacteria maybe critical for good health, but which microbial genera constitutes a "healthy microbiome" per se remains hotly debated. Both anatomical characteristics and breathing patterns seem to influence the access of microbes to the lungs [19]. Although the lungs are subjected to constant immigration from the oropharynx, their microbiome is distinctive to that of the upper respiratory tract which, in turn, displays greater similarities to that of the stomach [20]. Dickson et al. [21] proposed three models to explain maintenance of microbial homeostasis in health the modalities with which the homeostasis may be lost in disease.

The first model (Fig. 2) postulates that the community of species forming the LMt is determined by the balance of three factors: migration, elimination, and reproduction rates of microbiota as determined by regional growth conditions [21]. Migration to the LMt is at least in part attributable to micro-aspiration that has long been known to be frequent even among healthy subjects [21]. Bacteria elimination is commonly due to the mucociliary clearance, as well as the innate and adaptive immune defences. According to Dickson's conceptual model [21], factors involved in the reproduction rates of LMt include oxygen tension, $\mathrm{pH}$, blood perfusion, alveolar ventilation, temperature, and the concentration and activation of host inflammatory cells. 


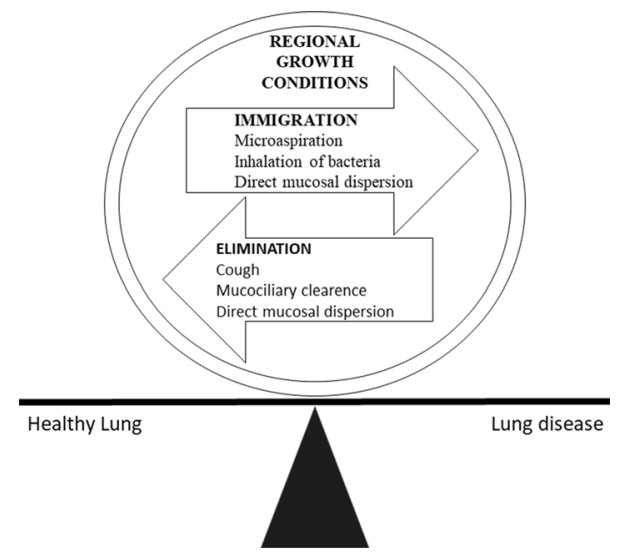

Fig. 2 Representation of the first Dickson's model. In health subjects, lungs microbial community is mainly determined by the balance of immigration (micro-aspiration) and elimination (cough, mucociliary clearance and immune defences). Microbial niches are a steady state of dynamic equilibrium, determined by the balance of immigration and elimination factors, influenced by anatomical, functional and clinical features

The second model implicates the availability of nutritional factors as a key determinant of all reproducing bacterial communities. Since in healthy conditions, the airway lumen mainly contains air, the availability of nutrients for most bacteria is relatively limited [22]; this fact might account for the minor role of local reproduction in the composition of the bacterial community in healthy subjects. On the other hand, the airways of patients with obstructive airway diseases, such as cystic fibrosis, chronic bronchitis, bronchiectasis, and asthma, contain a dense, protein-rich growth medium of secreted mucus [23]. Furthermore, in some clinical conditions, such as pneumonia and acute respiratory distress syndrome (ARDS), the alveoli are flooded with protein-rich oedema from an injured alveolar-capillary barrier. All these environments may contribute to the bacterial overgrowth and microbiota changes during lung diseases.

The final proposed mechanism is the so-called "signalling stress response", a molecular mechanism by which tissues and cells reciprocally communicate perturbations of the internal "milieu". Signalling molecules include hormones (e.g., glucocorticoids, oestrogens, and androgens), neurotransmitters (e.g., catecholamines and endogenous opioids) and cytokines (e.g., TNFs, IL-1, IL-6, and IL-8) [24, 25]. Recent data documented that some microbes can identify and adapt to the signaling molecules that human cells use to communicate [21].

\section{Gut microbiota and the gut-lung axis}

The human intestinal microbiota is one of the most densely populated microbial communities playing important

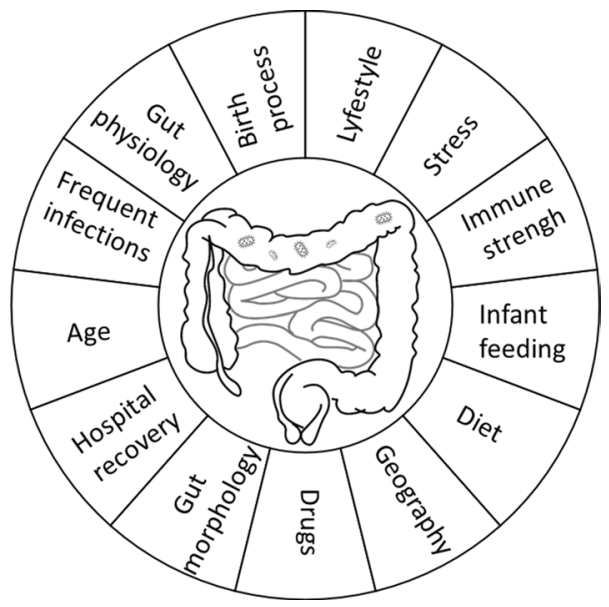

Fig. 3 Major factors influencing the development of gut microbiome

metabolic and protective roles in human health. The gut microbiota contains a plethora of bacteria, with most of them being obligate anaerobes from the phyla Actinobacteria, Verrucomicrobia, Firmicutes, Fusobacteria, Proteobacteria, and Bacteroidetes, Firmicutes, and Bacteroidetes represent about $90 \%$ of the gut microbiota [26]. It is hard to identify exactly what phyla characterize a microbiota, but it is even more difficult to know which bacterial genera make it up. For example, in the gut microbiota, Firmicutes phylum is composed of more than two hundred different genera (most frequently Bacillus, Lactobacillus, Enterococcus, Clostridium, and Ruminicoccus); on the other hand, the Bacterioidetes phylum mainly consists of genera represented by Prevotella and Bacterioides [27]. In an animal model of the human gut ecosystem, Turnbaugh et al. discovered that the gut microbiota of younger adults mice highly depends on their diet and lifestyle, suggesting a similar situation in human [28]. Accordingly, the later life stages have been associated with changes in gut microbiota due to several different factors such as those depicted in Fig. 3. Individual gut microbiota are characterized by clusters of bacteria called enterotypes [26]. There are three dominant bacteria clusters characterized, respectively, by Bacteroides in enterotype I, Prevotella in enterotype II, and Ruminococcus in enterotype III. Each enterotype harbours hundreds of bacteria genera [29]. Although a specific microbial population cannot define a unique healthy gut microbiome, the stability of bacteria genera in each enterotypes seems crucial in healthy status [29]. Indeed, bacteria in each enterotype that characterize individual gut microbiota remain stable from adulthood and can be modified by a process called "dysbiosis". Dysbiosis is a change in gut microbiota composition and may be the cause or consequence of disorders. It is hard to establish if a change in gut microbiome is beneficial or damaging. In health, the gut microbiome can prevent the attachment of 
exogenous pathogenic bacteria to the wall of the gastrointestinal tract and it also has direct bactericidal effects [30].

There is a clear cross-talk between the gut and the lungs, also called gut-lung axis, that is vital for maintaining homeostasis and educating the host immune system [31]. Interestingly, gut dysbiosis is also associated with lung disorders and infections, such as asthma and infections. Soluble microbial components and metabolites transported via the circulation, such as peptidoglycans or lipopolysaccharide (LPS), recognized by host cells may play a role in protection against asthma [32]. More precisely, reduction in genus Bifidobacteria and increase in genus Clostridia in the gut are associated with early life asthma [33]. Reciprocally, changes in lung microbial community can influence the gut microbiota composition, suggesting that gut and lung are linked organs [34]. In mice depleted of a microbiota, intrarectal LPS administration restored the capacity to mount $\mathrm{T}_{\mathrm{H}} 2$ responses, suggesting the ability of intestinal microbiotaderived LPS to affect pulmonary responses to allergens [35]. This is a bi-directional modulation; for instance, infection lung disease, such as pneumonia caused by Pseudomonas Aeruginosa, leads to a reduction in gut epithelial proliferation and blocks the $\mathrm{M}$ phase of cell cycle and induces dysbiosis [36]. Another example of the intricate relationship between gut microbiota and LMt is the possible relationship between lung function parameters and microbiome compositions in some pulmonary diseases [37]. Gut microbes help in assimilating nutrients that are indigestible by humans (i.e., fibres), and those whose metabolites can modulate gastrointestinal and lung immunity. As illustrated in Fig. 4, in vitro and in vivo studies have shown that nutritional factors are used by the gut microbiota bacteria to synthesize metabolites, including chain fatty acids (SCFAs), from soluble fibers [38]. The SCFAs (acetate, propionate, and butyrate) promote recruitment and maturation of immune cells, and therefore, they may represent a protective factor against inflammatory response [39]. Antigens are processed by gastro-intestinal tract dendritic cells that promote the proliferation and expansion of $\mathrm{T}$ cells, which, in turn reach various sites, where infection or antigen exposure occurred. Dysbiosis in the gut microbiota may, therefore, lead to impaired proliferation and expansion of T-cell subsets, as well as to increased inflammation contributing negatively on health and systemic immune response [40].

Another important link between gut and lungs is the migration of immune cells from the gut to the lungs by common mucosal immune system [41]. In brief, immune cells can migrate and colonise inductive sites forming the

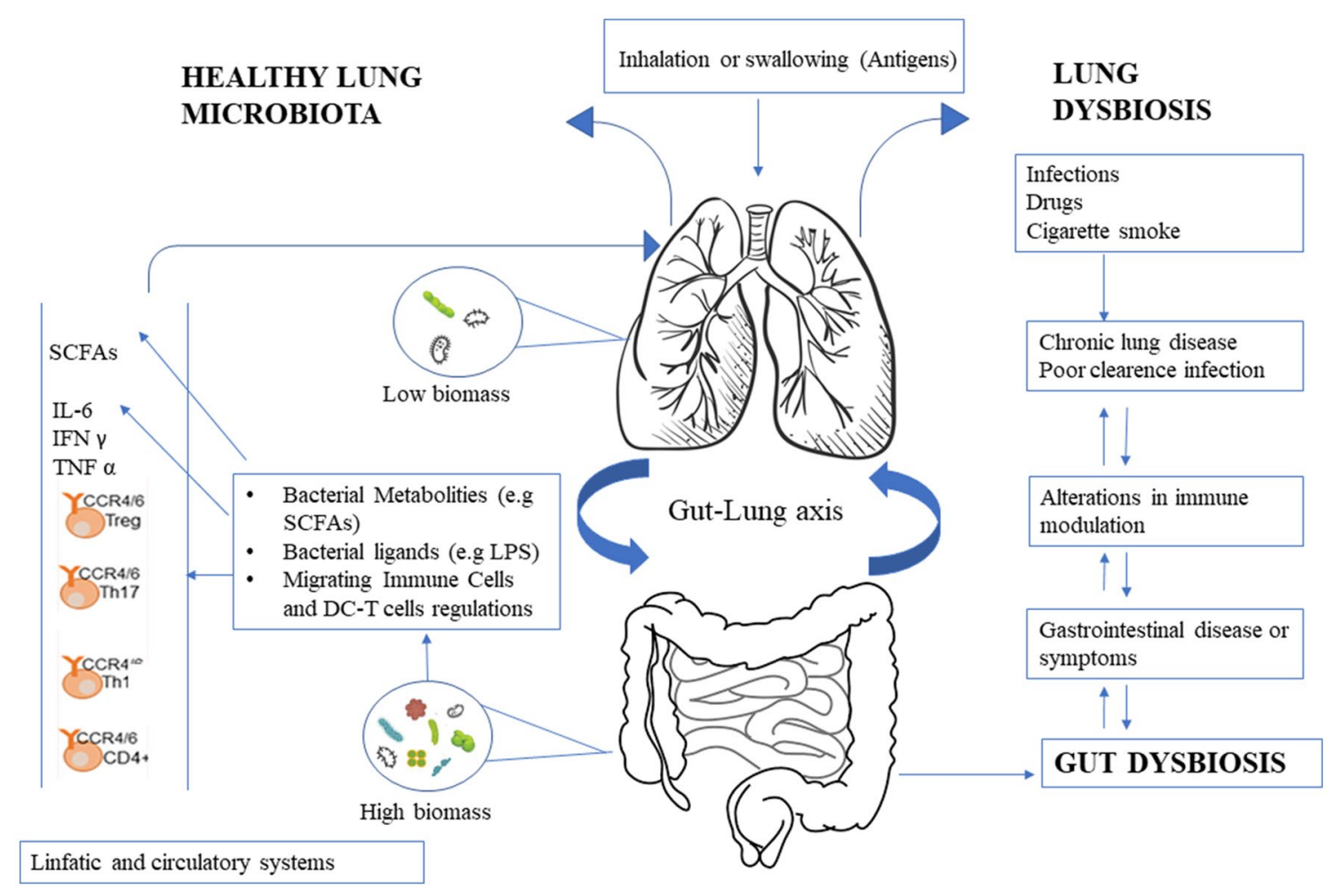

Fig. 4 Gut-lung axis. Gut and lung are linked organs that can reciprocally influence their homeostasis. The gut microbiota dysbiosis is associated with lung disorders and infections. SCFAs short-chain fatty acids, $L P S$ lipopolysaccharide, $D C-T$ cells dendritic cell-T cell, $I L-6$ interleukin-6, IFN $\gamma$ interferon-gamma, $T N F \alpha$ tumor necrosis factor alpha, CCR 4/6 CC chemokine receptor 4/6, CD 4+ cluster of differentiation antigen 4, Thl T-Helper Cell Type 1 
mucosa-associated lymphoid tissue, the gut-associated lymphoid tissue, and the nasopharyngeal-associated tissue; migration occurs via the lymphatic system [41]. Thus, the lymph may represent the connecting organ between the gut—where primary sensitization occurs—and the lung [41].

\section{Lung microbiota and respiratory diseases}

\section{Lung microbiota and smoke}

Relatively few studies have addressed the problem of microbiome density and variability in the airway and lungs of smoking subjects, so that most of current knowledge derives from data performed in animal models. Cigarette smoke contains thousands of chemical components including nitric oxide, carbon monoxide, nicotine, formaldehyde, acetone, ammonia, and acrolein. These substances contact the respiratory mucosa and are associated with chronic inflammation in smokers. Recently, Zhang et al. [42] studied that 40 mice exposed to tobacco smoke and observed that microbial diversity was higher in the smoking group, suggesting that smoke exposure increases the risk of bacterial infection, thereby increasing microbial diversity. The authors concluded that smoking influences microbial diversity and communities of the lower respiratory tract, suggesting that future studies on smoke-induced inflammation should also consider the LMt variation [42]. In recent years, it has been shown that the lungs of healthy smokers contain a bacterial microbiome that is quantitatively and qualitatively diverse from that of the oral cavity and extra-thoracic airways [43]. However, the role of microbial diversity remains unclear, since in contrast with Zhang et al. [42], human LMt diversity is often lower in subjects with poor lung function, and it is most commonly associated with dominance by Pseudomonas spp. The fact that some smokers, with spirometric evidence of lung disease, had a less different LMt compared with smokers, with normal lung function, and indicates that LMt changes can be an earlier sign of respiratory disease [43]. A novel study performed in smokers addressed the question of whether the LMt is related to the smoking status and to the development of ARDS in trauma patients [44]. Cigarette smoking is commonly associated with increased ARDS' risk in patients after severe trauma, but the underlying mechanism is unknown. In trauma patients with ARDS, Panzer et al. [44] documented that the smoking status was significantly associated with lung bacterial community composition at admission, particularly with a significant enrichment of potential pathogens, including Prevotella, Haemophilus, Streptococcus, Treponema, and Fusobacterium. Furthermore, ARDS development was characterised by relative enrichment of Enterobacteriaceae and of specific taxa enriched at baseline in smokers, including Prevotella and Fusobacterium.

\section{Microbiota and COPD}

Chronic obstructive pulmonary disease (COPD) is a heterogeneous disorder characterized by persistent respiratory symptoms, airflow obstruction, chronic airway inflammation, and frequent exacerbations [45]. COPD treatment is guided by the degree of airflow limitation or airway obstruction, but these variables alone may not suffice for optimal management [45]. In COPD, a multitude of bacteria, including potential respiratory pathogens, is often present. In addition, with increasing degrees of airflow limitation, there is also an increased recovery of opportunistic pathogens such as Pseudomonas aeruginosa [46]. This bacterial colonization correlates with the severity of inflammatory response, radiological changes, pathological variations of a local immune response, and increased daily symptoms [47]. As mentioned above, insults such as tobacco smoke, the major COPD cause, can impair innate immune defences and lead to a change in the abundance, taxonomic composition, and phylogenetic diversity of the LMt. In 2010, Hilty et al. [48] compared the microbiota of five patients with COPD to that of eleven subjects with asthma and eight healthy controls. They found that pathogenic Proteobacteria, particularly Haemophilus, were more frequent in asthmatics and COPD than in controls. Conversely, Bacteroidetes, particularly Prevotella, were less frequent in asthmatics and COPD. Erb-Downward et al. [43] compared BAL fluid from 3 cohorts of subjects: smokers without airflow obstruction, subjects with obstruction, and healthy subjects as control. They observed similar concentrations of 16S rRNA genes across groups, suggesting a similar range of bacterial burdens across groups, but decreased diversity in individuals with the most severe airflow limitation [43]. Finally, Sze et al. [49] examined the LMt in five explanted lungs from COPD patients with severe airflow obstruction and four healthy donor lungs. Dysbiosis in LMt, in particular expansion of Proteobacteria and Actinobacteria phyla in COPD patients and in Bacteroidetes and Firmicutes in the control group, was associated with increased alveolar destruction and greater immune cells' infiltration. These authors [50] also examined the LMt profiles linking with micro-computed tomography, quantitative histology, and host gene expression data from lung explants of COPD patients with severe lung disease. The presence of emphysema and airway remodelling was associated with decreased bacterial diversity, which negatively correlated with $\mathrm{CD} 4^{+} \mathrm{T}$ cell infiltration. Members of the Proteobacteria and Actinobacteria phyla were found to be more abundant in COPD versus control tissue samples, whereas Firmicutes and Bacteroidetes were less prevalent in emphysematous lung areas [50]. The observed microbiota 
compositional differences were also associated with infiltrating numbers of eosinophils, neutrophils and B cells as well as a number of differentially expressed genes, suggesting that host-immune responses correlated with the lung dysbiosis [50]. Wang et al. [51] studied sputum samples of 87 subjects with COPD collected during stability, exacerbation, 2 and 6 week post-therapy; dysbiosis in LMt appeared to be associated with exacerbation events and indicative of two specific exacerbation phenotypes (bacterial and eosinophilic) [51]. In the "bacterial" phenotype, Proteobacteria was increased, while in "eosinophilic" phenotype, an increase in Firmicutes was observed. In an individual COPD patient, LMt changes may represent a potential biomarker, as well as a therapeutic intervention target for COPD [51]. A different study [52] analyzed sputum samples from 102 patients, hospitalized due to acute COPD exacerbation (AECOPD) and followed up all patients for 1 year after discharge. The authors suggest that the sputum dysbiosis in AECOPD is associated with the 1-year mortality and may identify subjects with a poor prognosis at the hospitalization time.

Finally, the AERIS (Acute Exacerbation and Respiratory InfectionS) study in COPD patients analysed the LMt at both stable and exacerbation time points in 584 sputum samples from 101 patients with COPD over 1 year [53]. LMt dysbiosis in COPD patients correlated with disease severity and exacerbations. Exacerbations within individuals showed higher microbiome variability than at stable time points, and patients with frequent exacerbations had significant changes in LMt patterns. The AERIS study confirmed the association of some bacterial genera, with disease severity, frequent exacerbation events and presence of bronchiectasis. This study may help to identify lung bacterial phenotypes and biomarkers that may be used for classification of COPD phenotypes and facilitate appropriate treatments [53].

Taken together, the result of studies performed so far on COPD patients seem to indicate that characterisation of the microbiome may provide important, additional information regarding COPD phenotypes, the inflammatory degree of and immunological dysfunction, as well as the susceptibility to disease exacerbations and progression.

\section{Microbiota impact in asthma}

Emerging and increasing data suggest that the microbiome may play a crucial role in the asthma development [54]. Asthma is a disease that often presents with a variety of clinical features and different patterns of immune responses, likely reflecting diverse genetic and environmental components [54]. Reduced lower gut microbiota diversity in early life seems to correlate with the occurrence of asthma [55]. An association between a lower numbers of some bacteria genera (Bifidobacteria, Akkermansia and Faecalibacterium) and higher risk of developing atopy and/or early life asthma
[56]. In addition, the impairment of gut microbiota balance, resulted by continuative antibiotic use, increases the risk of asthma [57]. According with the so-called hygiene theory [58], reduced exposure to microbes in urban areas is associated with a higher incidence of allergy and asthma Canadian children at asthma risk shows decreased gut colonies of Lachnospira, Veillonella, Faecalibacterium, and Rothia [59]. On the contrary, inoculations of these bacteria in germfree mice have a protective effect against airway inflammation and asthma [60]. An analysis of bronchoalveolar lavage (BAL) from children with severe asthma has shown abundance of Proteobacteria (mainly Haemophilus), Firmicutes Staphylococcus and Streptococcus), Bacteroidetes (mainly Prevotella), and Actinobacteria. In detail, Staphylococcus and Haemophilus were more abundant in asthmatic children than control subjects, the latter showing abundance of Prevotella [48].

\section{Idiopathic pulmonary fibrosis and microbiota}

Most of our knowledge to date regarding the LMt impact on interstitial lung diseases mainly relates to the idiopathic pulmonary fibrosis (IPF), where environmental exposures, gastro-oesophageal reflux, and microbial agents have been hypothesized to initiate the aberrant healing response [61]. In IPF lungs, there is an increased bacterial burden and overrepresentation of potential pathogens associated with disease exacerbations and progression [62]. In addition, a specific host immune response to an altered LMt is associated with the prognosis [63]. Since antibiotic administration or immunization against pathogens may improve IPF outcomes, the LMt has recently been proposed as a potential therapeutic target [64]. Han and colleagues showed that Veillonella, Prevotella, and Cronobacter species were the most prevalent in IPF patients; furthermore, the presence of abundant Streptococcus or Staphylococcus sequences were associated with a clinically significant reduction in progression-free survival time [62]. A prospective study, comparing microbial composition in bronchoalveolar lavage fluid of IPF patients and normal controls, observed that IPF patients had significantly higher bacterial burden [65]. Among IPF patients, a higher bacterial load was associated with a significantly reduced progression-free survival time, an effect independent of age and smoking status [64].

However, much more studies are required.

\section{Microbiota and lung infections}

According to Dickson's model of lung biogeography, even healthy lungs are subjected to constant immigration from oropharyngeal microbes via micro-aspiration [66]; this immigration is thought to be regulated by the dynamics of the aerodigestive tract and may, therefore, be boosted 
in conditions of reduced level of consciousness, during mechanical ventilation and by endotracheal intubation. As a result, a state of increased immigration, decreased elimination, and favourable growth conditions for potential pathogens can ensue [67]. Any source of alveolar injury and inflammation can trigger a cascade of effects eventually leading to increased concentrations of intra-alveolar catecholamines, which in turn can promote the growth and virulence of select bacterial communities and perpetuate alveolar inflammation [68]. Ecologically, infections are characterised by an increase in microbial burden and a decrease in community diversity, along with an increased host inflammation and tissue injury. Bacterial pneumonia exemplifies these features: it is characterised by increased bacterial burden and low community diversity [69].

\section{Microbiota and lung cancer}

The role of microbiome in lung cancer is ill defined. Since the inflammatory responses is linked with the lung cancer development [70], some studies documented that infections such as tuberculosis or pneumonia increase the risk of lung cancer [71]. Although some discrepancies exist, two bacterial genera (Veillonella and Megasphaera) have been found to be increased significantly in lung cancer patients [72]; Yan et al. [73] demonstrated that the salivary microbiome was different in lung cancer patients compared with controls, suggesting the salivary microbiome as a potential bacterial biomarker of lung cancer.

The limited studies, evaluating the microbiome in lung cancer, their small sample sizes, and the range of sampling techniques used, prevent any firm conclusions being made.

\section{Therapeutic implications}

Much remains to be learned on if and how the microbiome can therapeutically be targeted to improve the outcomes of respiratory disorders. A diet rich in fibre simultaneously changes the intestinal and LMt, indicating diet influence on lung immunity [74]. Nutrition and administration of probiotics could be a valid strategy to maintain or restore a functional microbiome [74]. Fibre intake increases SCFAs' levels in blood and has been reported both to provide protection against allergic inflammation in lung and to reduce mortality from respiratory disease [39]. Studies on mice models [75] indicate that the administration of probiotic bacteria-such as Lactobacillus rhamnosus, Bifidobacterium lactis, and Bifidobacterium breve, which are gut microbiota components-suppresses allergic and autoimmune responses, reduces allergic symptoms, and inhibits allergic airway response [76]. Some evidence suggests beneficial effects of probiotics on human immune responses [76]; however, data from the literature are not fully consistent and discrepancies have been found regarding probiotic effectiveness [77]. In addition, the dietary intake has been shown to modify systemic inflammation in asthma, particularly in the neutrophilic inflammatory phenotype [33]. As food fibers are used by gut bacteria to biosynthesize SCFAs [78], higher intakes of fruits and vegetables may have a positive impact on asthma risk and control. In fact, recently, the European Academy of Allergy and Clinical Immunology recommended in clinical practice to increase the net intake of fruits and vegetables to decrease the asthma risk, especially in children [79]. Dietary intervention should, therefore, be incorporated into the routine clinical management of patients with asthma, to achieve overall health benefits and disease management [79]. In vitro studies of cigarette smoke induced diseases, such as COPD, indicate that the administration of Lactobacillus rhamnosus and Bifdobacterium breve abolishes the release of pro-inflammatory mediators by the macrophages in response to cigarette smoke [75]. Moreover, probiotics may restore natural killer cell activity, which is depressed in smokers [80]. Oral feeding of the Lactobacillus acidophilus to mice lung cancer model, receiving cisplatin treatment, shows reduction in tumour size and higher survival rate [81]. Interestingly, Daillère R. et al. showed better outcomes in advanced lung cancer patients when Enterococcus hirae and Barnesiella intestinihominis were administered in combination with chemoimmunotherapy possibly due to an improved immunomodulatory action by these probiotics [82]. Attention should also be given to identify the detrimental effects of some of the current therapies (such as inhaled and systemic corticosteroid and antibiotics) for lung diseases on the functionality of the microbiota. The current anti-AECOPD treatments are known to impact on LMt and, especially with antibiotics and oral steroids, their effect may persist well after discontinuation [83]. Antibiotics exert sustained suppressive effects, whereas steroids increase relative abundance of many bacterial communities, such as members of the Proteobacteria phylum (Moraxellaceae, Pasteurellaceae, Pseudomonadaceae, and Enterobacteriaceae) [83]. Furthermore, patients treated with both antibiotics and steroids showed a significant increase in Proteobacteria abundance. Conceivably, drug-induced phenotypical alterations in LMt contribute to the frequency and severity of exacerbations, especially with prolonged therapies. Current guidelines recommend the use of inhaled corticosteroids (ICS) for asthma to control airway inflammation [84]. The ICS' impact on the airway microbiome in asthma is unclear. Chronic use of ICS seems to be associated with a greater richness and diversity in microorganism samples compared with samples of non-exposed subjects [83]. In patients with mild-to-moderate asthma, irrespective of the ICS' use, Proteobacteria is the dominant phylum, suggesting that airway dysbiosis may be a feature of asthma itself and is not solely attributable to ICS therapy. Indeed, a 
recent study confirmed that the diversity and composition of the bronchial microbiome was not influenced by ICS' treatment alone but rather by the combination therapy of oral and ICS' treatment [85].

\section{Conclusions}

Growing evidence indicates that the LMt may have an important role in lung health and disorders. LMt modifications characterise chronic lung diseases and may account for exacerbations caused by endogenous microbiota alterations and susceptibility to new infections. Much remains to be done via adequate nutritional and/or pharmacological interventions in attempt to restore a healthy microbiota in pulmonary and non-pulmonary diseases, whose origin and clinical outcomes appear to be strongly influenced by the microbiota. We think that the fine evaluation of gut and LMt represents a new and stimulating research field with theoretically unlimited prospective and may be in the future, it will be a key therapeutic target for the prevention and treatment of critical lung illness.

Funding The study was unfunded.

\section{Compliance with ethical standards}

Conflict of interest The authors declare no conflict of interest related to the present manuscript.

Statement of human and animal rights This article does not contain any studies with animals performed by any of the authors.

Informed consent All participants provided informed consent prior to their participation.

\section{References}

1. Lederberg BJ, McCray AT (2001) 'Ome sweet' omics—a genealogical treasury of words. Sci 15:8. https://doi.org/10.1110/ ps.9.11.2181

2. Marchesi JR, Ravel J (2015) The vocabulary of microbiome research: a proposal. Microbiome 3:31. https://doi.org/10.1186/ s40168-015-0094-5

3. Yatsunenko T, Rey FE, Manary MJ et al (2012) Human gut microbiome viewed across age and geography. Nature 486:222-227. https://doi.org/10.1038/nature11053

4. Kumar M, Babaei P, Ji B, Nielsen J (2016) Human gut microbiota and healthy aging: recent developments and future prospective. Nutr Health Age 4:3-16. https://doi.org/10.3233/NHA-150002

5. Laterza L, Rizzatti G, Gaetani E et al (2016) The gut microbiota and immune system relationship in human graft-versus-host disease. Mediterr J Hematol Infect Dis 8:e2016025-e2016025. https ://doi.org/10.4084/MJHID.2016.025

6. Ames NJ, Ranucci A, Moriyama B, Wallen GR (2017) The human microbiome and understanding the 16S rRNA gene in translational nursing science. Nurs Res 66:184-197. https://doi. org/10.1097/NNR.0000000000000212

7. Faner R, Sibila O, Agustí A et al (2017) The microbiome in respiratory medicine: current challenges and future perspectives. Eur Respir J 49:1-12. https://doi.org/10.1183/13993003.02086-2016

8. Budden KF, Shukla SD, Rehman SF et al (2019) Functional effects of the microbiota in chronic respiratory disease. Lancet Respir Med 2600:1-14. https://doi.org/10.1016/S2213-2600(18)30510-1

9. Ahmed B, Cox MJ, Cuthbertson L (2019) Growing up with your airway microbiota: a risky business. Thorax 74:525 LP-526. https ://doi.org/10.1136/thoraxjnl-2019-213162

10. Niccolai E, Boem F, Russo E, Amedei A (2019) The gut-brain axis in the neuropsychological disease model of obesity: a classical movie revised by the emerging director "microbiome". Nutrients 11:156. https://doi.org/10.3390/nu11010156

11. Amedei A, Boem F (2018) I've gut a feeling: microbiota impacting the conceptual and experimental perspectives of personalized medicine. Int J Mol Sci 19:3756. https://doi.org/10.3390/ijms1 9123756

12. Russo E, Bacci G, Chiellini C et al (2018) Preliminary comparison of oral and intestinal human microbiota in patients with colorectal cancer: a pilot study. Front Microbiol 8:2699. https://doi. org/10.3389/fmicb.2017.02699

13. Beck JM, Young VB, Huffnagle GB (2012) The microbiome of the lung. Transl Res 160:258-266. https://doi.org/10.1016/j. trs1.2012.02.005

14. Cui L, Morris A, Huang L et al (2014) The microbiome and the lung. Ann. Am. Thorac, Soc

15. Dickson RP, Erb-Downward JR, Prescott HC et al (2015) Intraalveolar catecholamines and the human lung microbiome. Am J Respir Crit Care Med 192:257-259. https://doi.org/10.1164/ rccm.201502-0326LE

16. Charlson ES, Bittinger K, Haas AR et al (2011) Topographical continuity of bacterial populations in the healthy human respiratory tract. Am J Respir Crit Care Med 184:957-963. https://doi. org/10.1164/rccm.201104-0655OC

17. Lemon KP, Klepac-Ceraj V, Schiffer HK et al (2010) Comparative analyses of the bacterial microbiota of the human nostril and oropharynx. MBio 1:e00129-e210. https://doi.org/10.1128/ mBio.00129-10

18. Segal LN, Alekseyenko AV, Clemente JC et al (2013) Enrichment of lung microbiome with supraglottic taxa is associated with increased pulmonary inflammation. Microbiome 1:19. https://doi. org/10.1186/2049-2618-1-19

19. Mathieu E, Escribano-Vazquez U, Descamps D et al (2018) Paradigms of lung microbiota functions in health and disease, particularly, in asthma. Front Physiol 9:1-11. https://doi.org/10.3389/ fphys.2018.01168

20. Bassis CM, Erb-Downward JR, Dickson RP et al (2015) Analysis of the upper respiratory tract microbiotas as the source of the lung and gastric microbiotas in healthy individuals. MBio 6

21. Dickson RP, Erb-Downward JR, Huffnagle GB (2015) Homeostasis and its disruption in the lung microbiome. Am J Physiol Lung Cell Mol Physiol. https://doi.org/10.1152/ajplung.00279.2015

22. Kasubuchi M, Hasegawa S, Hiramatsu T et al (2015) Dietary gut microbial metabolites, short-chain fatty acids, and host metabolic regulation. Nutrients 7:2839-2849. https://doi.org/10.3390/nu704 2839

23. Günther A, Siebert C, Schmidt R et al (1996) Surfactant alterations in severe pneumonia, acute respiratory distress syndrome, and cardiogenic lung edema. Am J Respir Crit Care Med 153:176-184. https://doi.org/10.1164/ajrccm.153.1.8542113

24. Zhang X, Essmann M, Burt ET, Larsen B (2000) Estrogen effects on Candida albicans: a potential virulence-regulating mechanism. J Infect Dis 181:1441-1446 
25. Zaborina O, Lepine F, Xiao G et al (2007) Dynorphin activates quorum sensing quinolone signaling in Pseudomonas aeruginosa. PLoS Pathog 3:e35-e35. https://doi.org/10.1371/journ al.ppat.0030035

26. Arumugam M, Raes J, Pelletier E et al (2011) Enterotypes of the human gut microbiome. Nature 473:174-180. https://doi. org/10.1038/nature09944

27. Papa E, Docktor M, Smillie C et al (2012) Non-invasive mapping of the gastrointestinal microbiota identifies children with inflammatory bowel disease. PLoS ONE 7:e39242

28. Turnbaugh PJ, Ridaura VK, Faith JJ, et al (2009) The Effect of Diet on the Human Gut Microbiome: A Metagenomic Analysis in Humanized Gnotobiotic Mice. Sci Transl Med 1:6ra14 LP-6ra14

29. Rinninella E, Raoul P, Cintoni M et al (2019) What is the healthy gut microbiota composition? A changing ecosystem across age, environment, diet, and diseases. Microorganisms 7:14. https://doi. org/10.3390/microorganisms7010014

30. Bull MJ, Plummer NT (2014) Part 1: the human gut microbiome in health and disease. Integr Med (Encinitas) 13:17-22

31. Dang AT, Marsland BJ (2019) Microbes, metabolites, and the gutlung axis. Mucosal Immunol 12:843-850. https://doi.org/10.1038/ s41385-019-0160-6

32. Wypych TP, Wickramasinghe LC, Marsland BJ (2019) The influence of the microbiome on respiratory health. Nat Immunol. https ://doi.org/10.1038/s41590-019-0451-9

33. Kalliomäki M, Kirjavainen P, Eerola E et al (2001) Distinct patterns of neonatal gut microflora in infants in whom atopy was and was not developing. J Allergy Clin Immunol 107:129-134. https ://doi.org/10.1067/mai.2001.111237

34. Ichinohe T, Pang IK, Kumamoto Y, et al (2011) Microbiota regulates immune defense against respiratory tract influenza A virus infection. Proc Natl Acad Sci 108:5354 LP-5359

35. Qian G, Jiang W, Zou B et al (2018) LPS inactivation by a host lipase allows lung epithelial cell sensitization for allergic asthma. J Exp Med 215:2397-2412. https://doi.org/10.1084/jem.20172225

36. Coopersmith C, Stromberg P, Davis G et al (2003) Sepsis from Pseudomonas aeruginosa pneumonia decreases intestinal proliferation and induces gut epithelial cell cycle arrest. Crit Care Med 31:1630-1637. https://doi.org/10.1097/01.CCM.0000055385 .29232 .11

37. Burke DG, Fouhy F, Harrison MJ et al (2017) The altered gut microbiota in adults with cystic fibrosis. BMC Microbiol 17:58. https://doi.org/10.1186/s12866-017-0968-8

38. S Anand SS Mande 2018 Diet front microbiol microbiota and gut-lung connection 10.3389/fmicb.2018.02147

39. Vinolo MAR, Rodrigues HG, Nachbar RT, Curi R (2011) Regulation of inflammation by short chain fatty acids. Nutrients 3:858876. https://doi.org/10.3390/nu3100858

40. Samuelson DR, Welsh DA, Shellito JE (2015) Regulation of lung immunity and host defense by the intestinal microbiota. Front Microbiol 6:1085. https://doi.org/10.3389/fmicb.2015.01085

41. McGhee JR, Fujihashi K (2012) Inside the mucosal immune system. PLOS Biol 10:e1001397

42. Zhang R, Chen L, Cao L et al (2018) Effects of smoking on the lower respiratory tract microbiome in mice. Respir Res 19:253. https://doi.org/10.1186/s12931-018-0959-9

43. Erb-Downward JR, Thompson DL, Han MK et al (2011) Analysis of the lung microbiome in the "healthy" smoker and in COPD. PLoS ONE 6:e16384-e16384. https://doi.org/10.1371/journ al.pone. 0016384

44. Panzer AR, Lynch SV, Langelier C et al (2017) Lung microbiota is related to smoking status and to development of acute respiratory distress syndrome in critically ill trauma patients. Am J Respir Crit Care Med 197:621-631. https://doi.org/10.1164/rccm.20170 2-0441OC
45. Global Initiative for Chronic Obstructive Lung Disease (2019) GOLD Report 2019. 1-155

46. Di Stefano A, Ricciardolo FLM, Caramori G, et al (2017) Bronchial inflammation and bacterial load in stable COPD is associated with TLR4 overexpression. Eur Respir J 49:

47. Langille MGI, Zaneveld J, Caporaso JG et al (2013) Predictive functional profiling of microbial communities using 16S rRNA marker gene sequences. Nat Biotechnol 31:814-821. https://doi. org/10.1038/nbt.2676

48. Hilty M, Burke C, Pedro H et al (2010) Disordered microbial communities in asthmatic airways. PLoS ONE 5:e8578-e8578. https://doi.org/10.1371/journal.pone.0008578

49. Sze MA, Dimitriu PA, Hayashi S et al (2012) The lung tissue microbiome in chronic obstructive pulmonary disease. Am J Respir Crit Care Med 185:1073-1080. https://doi.org/10.1164/ rccm.201111-2075OC

50. Sze MA, Dimitriu PA, Suzuki M et al (2015) Host response to the lung microbiome in chronic obstructive pulmonary disease. Am J Respir Crit Care Med 192:438-445. https://doi.org/10.1164/ rccm.201502-0223OC

51. Wang L, Hao K, Yang T, Wang C (2017) Role of the lung microbiome in the pathogenesis of chronic obstructive pulmonary disease. Chin Med J (Engl) 130(17):2107-2111. https://doi. org/10.4103/0366-6999.211452

52. Leitao Filho FS, Alotaibi NM, Ngan D et al (2018) Sputum microbiome is associated with 1-year mortality following COPD hospitalizations. Am J Respir Crit Care Med. https://doi.org/10.1164/ rccm.201806-1135OC

53. Mayhew D, Devos N, Lambert C et al (2018) Longitudinal profiling of the lung microbiome in the AERIS study demonstrates repeatability of bacterial and eosinophilic COPD exacerbations. Thorax 73:422-430. https://doi.org/10.1136/thoraxjnl-2017210408

54. Sullivan A, Hunt E, MacSharry J, Murphy DM (2016) The microbiome and the pathophysiology of asthma. Respir Res 17:163. https://doi.org/10.1186/s12931-016-0479-4

55. Stiemsma LT, Turvey SE (2017) Asthma and the microbiome: defining the critical window in early life. Allergy Asthma Clin Immunol 13:3. https://doi.org/10.1186/s13223-016-0173-6

56. Fujimura KE, Sitarik AR, Havstad S et al (2016) Neonatal gut microbiota associates with childhood multisensitized atopy and T cell differentiation. Nat Med 22:1187

57. Stokholm J, Blaser MJ, Thorsen J et al (2018) Maturation of the gut microbiome and risk of asthma in childhood. Nat Commun 9:141. https://doi.org/10.1038/s41467-017-02573-2

58. Olszak T, An D, Zeissig S, et al (2012) Microbial exposure during early life has persistent effects on natural killer $t$ cell function. Science 80(336):489 LP-493

59. Dales RE, Zwanenburg H, Burnett R, Franklin CA (1991) Respiratory health effects of home dampness and molds among canadian children. Am J Epidemiol 134:196-203

60. Arrieta M-C, Stiemsma LT, Dimitriu PA, et al (2015) Early infancy microbial and metabolic alterations affect risk of childhood asthma. Sci Transl Med 7:307ra152 LP-307ra152

61. Raghu G, Collard HR, Egan JJ et al (2011) An official ATS/ ERS/JRS/ALAT statement: idiopathic pulmonary fibrosis: evidence-based guidelines for diagnosis and management. Am J Respir Crit Care Med 183:788-824. https://doi.org/10.1164/ rccm.2009-040GL

62. Han MK, Zhou Y, Murray S et al (2014) Lung microbiome and disease progression in idiopathic pulmonary fibrosis: an analysis of the COMET study. Lancet Respir Med 2:548-556. https://doi. org/10.1016/S2213-2600(14)70069-4

63. Salisbury ML, Han MK, Dickson RP, Molyneaux PL (2017) Microbiome in interstitial lung disease: from pathogenesis to 
treatment target. Curr Opin Pulm Med 23:404-410. https://doi. org/10.1097/MCP.0000000000000399

64. Molyneaux PL, Cox MJ, Willis-Owen SAG et al (2014) The role of bacteria in the pathogenesis and progression of idiopathic pulmonary fibrosis. Am J Respir Crit Care Med 190:906-913. https ://doi.org/10.1164/rccm.201403-0541OC

65. Molyneaux PL, Maher TM (2013) The role of infection in the pathogenesis of idiopathic pulmonary fibrosis. Eur Respir Rev 22:376 LP-381

66. Dickson RP, Erb-Downward JR, Freeman CM et al (2015) Spatial variation in the healthy human lung microbiome and the adapted island model of lung biogeography. Ann Am Thorac Soc 12:821830. https://doi.org/10.1513/AnnalsATS.201501-029OC

67. Zakharkina T, Martin-Loeches I, Matamoros S, et al (2017) The dynamics of the pulmonary microbiome during mechanical ventilation in the intensive care unit and the association with occurrence of pneumonia. Thorax 72:803 LP-810. https://doi. org/10.1136/thoraxjnl-2016-209158

68. Flierl MA, Rittirsch D, Nadeau BA et al (2007) Phagocyte-derived catecholamines enhance acute inflammatory injury. Nature 449:721

69. Dickson RP, Erb-Downward JR, Prescott HC et al (2014) Analysis of culture-dependent versus culture-independent techniques for identification of bacteria in clinically obtained bronchoalveolar lavage fluid. J Clin Microbiol 52:3605-3613. https://doi. org/10.1128/JCM.01028-14

70. Cho WCS, Kwan CK, Yau S et al (2011) The role of inflammation in the pathogenesis of lung cancer. Expert Opin Ther Targets 15:1127-1137. https://doi.org/10.1517/14728222.2011.599801

71. Mejri I, Ourari B, Cherif H, et al (2016) Pulmonary tuberculosis and lung cancer: A complex interaction. Eur Respir J 48

72. Lee SH, Sung JY, Yong D et al (2016) Characterization of microbiome in bronchoalveolar lavage fluid of patients with lung cancer comparing with benign mass like lesions. Lung Cancer 102:8995. https://doi.org/10.1016/j.lungcan.2016.10.016

73. Yan X, Yang M, Liu J et al (2015) Discovery and validation of potential bacterial biomarkers for lung cancer. Am J Cancer Res 5:3111-3122

74. Toh ZQ, Anzela A, Tang M, Licciardi P (2012) Probiotic therapy as a novel approach for allergic disease. Front Pharmacol 3:171

75. Mortaz E, Adcock IM, Ricciardolo FLM, et al (2015) in vitro administration of L. rhamnosus and B. breve suppresses the proinflammatory mediators induced by exposure of macrophages to cigarette smoke. These findings may indicate importance of probiotics in treatment of cigarette smoke induced diseases lik. PLoS One 10:e0136455

76. Zuccotti G, Meneghin F, Aceti A et al (2015) Probiotics for prevention of atopic diseases in infants: systematic review and metaanalysis. Allergy 70:1356-1371. https://doi.org/10.1111/all.12700

77. Lerner A, Shoenfeld Y, Matthias T (2019) Probiotics: if it does not help it does not do any harm. Really? Microorganisms 7:104. https://doi.org/10.3390/microorganisms7040104

78. Cummings JH, Macfarlane GT, Englyst HN (2001) Prebiotic digestion and fermentation. Am J Clin Nutr 73:415s-420s. https ://doi.org/10.1093/ajcn/73.2.415s

79. Garcia-Larsen V, Del Giacco SR, Moreira A et al (2016) Asthma and dietary intake: an overview of systematic reviews. Allergy 71:433-442. https://doi.org/10.1111/all.12800

80. Shi LH, Balakrishnan K, Thiagarajah K, et al (2016) Beneficial Properties of Probiotics. Trop life Sci Res 27:73-90. https://doi. org/10.21315/tlsr2016.27.2.6

81. Sivan A, Corrales L, Hubert N et al (2015) Commensal Bifidobacterium promotes antitumor immunity and facilitates anti-PD-L1 efficacy. Science 350:1084-1089. https://doi.org/10.1126/scien ce.aac4255

82. Daillère R, Vétizou $M$, Waldschmitt $\mathrm{N}$ et al (2016) Enterococcus hirae and Barnesiella intestinihominis facilitate cyclophosphamide-induced therapeutic immunomodulatory effects. Immunity 45:931-943. https://doi.org/10.1016/j.immuni.2016.09.009

83. Huang YJ, Sethi S, Murphy T et al (2014) Airway microbiome dynamics in exacerbations of chronic obstructive pulmonary disease. J Clin Microbiol 52:2813-2823. https://doi.org/10.1128/ JCM.00035-14

84. Becker AB, Abrams EM (2017) Asthma guidelines : the Global Initiative for Asthma in relation to national guidelines. 99-103. https://doi.org/10.1097/ACI.0000000000000346

85. Denner DR, Sangwan N, Becker JB et al (2016) Corticosteroid therapy and airflow obstruction influence the bronchial microbiome, which is distinct from that of bronchoalveolar lavage in asthmatic airways. J Allergy Clin Immunol 137:1398-1405.e3. https://doi.org/10.1016/j.jaci.2015.10.017

Publisher's Note Springer Nature remains neutral with regard to jurisdictional claims in published maps and institutional affiliations. 